\title{
Mathematical Models for the Influence of Cytarabine on White Blood Cell Dynamics in Acute Myeloid Leukemia
}

\author{
Felix Jost ${ }^{1 *}$, Enrico Schalk ${ }^{2}$, Kristine Rinke ${ }^{1}$, Thomas Fischer ${ }^{2}$, Sebastian Sager ${ }^{1}$ \\ 1 Institute of Mathematical Optimization, Faculty of Mathematics, \\ Otto-von-Guericke University Magdeburg, Germany \\ 2 Department of Hematology and Oncology, Medical Faculty, \\ Otto-von-Guericke University Magdeburg, Germany \\ * felix.jost@ovgu.de
}

\section{Abstract}

We investigate the personalisation and prediction accuracy of mathematical models for white blood cell (WBC) count dynamics during consolidation treatment using intermediate or high-dose cytarabine (Ara-C) in acute myeloid leukemia (AML). Ara-C is the clinically most relevant cytotoxic agent for AML treatment.

We extend the gold-standard model of myelosuppression and a pharmacokinetic model of Ara-C with different hypotheses of Ara-C's pharmacodynamic effects. We cross-validate 12 mathematical models using dense WBC count measurements from 23 AML patients. Surprisingly, the prediction accuracies are similarly good despite different modelling hypotheses. Therefore, we compare average clinical and calculated WBC recovery times for different Ara-C schedules as a successful methodology for model discrimination. As a result, a new hypothesis of a secondary pharmacodynamic effect on the proliferation rate seems plausible. Furthermore, we demonstrate how personalized predictions of the impact of treatment timing on subsequent nadir values could be used for clinical decision support.

\section{Author summary}

The major obstacle in accurately predicting the outcome of a medical therapy is the vast variation in individual response patterns. It concerns both the subjective experience of the patient and the objectively measurable achievement of a clinical remission with restoration of normal blood counts. Here, we address acute myeloid leukemia (AML)-chemotherapy using cytarabine (Ara-C) as this drug is this most important component of AML-treatment. In addition to the wide spectrum of genetic aberrations involved in pathogenesis leading to variations in patient response patterns, another facet of personalised medicine awaits exploration of its full potential: a systematic, mathematical approach to understand and manipulate the dynamics of relevant biomarkers. We use personalised mathematical models to describe and predict white blood cell (WBC) counts during AML consolidation treatment. We analyse why and to what extent low WBC counts, a serious adverse event during therapy, occur. In a comprehensive approach we investigate published models, compare them with our extended models and outline the impact of modelling assumptions and varying chemotherapy schedules on prediction accuracy and model discrimination. Our numerical results confirm the clinical finding that a newly proposed schedule is superior with respect to WBC recovery and shed new light on the reasons why. 


\section{Preamble on terminology and potentially confusing synonyms}

Our work is located in the intersection of mathematics, control theory, systems biology, pharmacology, and medicine. Words like "model" or "parameter" have different meanings in these scientific communities, and similar concepts have different names like "calibration", "estimation", or "personalisation". For convenience, we list some synonyms that we did (not) use in Table $\mathbf{S 1}$.

\section{Introduction}

Acute myeloid leukemia (AML) is a malignant clonal disorder of myeloid stem and progenitor cells. In untreated AML, immature neoplastic myeloid blasts rapidly proliferate and suppress the generation and maturation of blood cells in the bone marrow. While being a curable disease using chemotherapy including anthracyclines and/or cytarabine (Ara-C), this approach leads to prolonged myelosuppression with extremely low white blood cell (WBC) counts (leukopenia), i.e. values below $1 G / L$, associated with a high risk of infection and treatment-related mortality [1].

Consolidation treatment, repetitive (up to 4) cycles of intermediate-/high-dose Ara-C $\left(1-3 g / m^{2}\right)[2]$, is given once patients achieve complete remission (CR) and is considered the most important part of chemotherapy in preventing relapses. The current standard treatment of $3 \mathrm{~g} / \mathrm{m}^{2}$ Ara-C infusion lasting 3 hours every 12 hours on days 1,3 and 5 for patients aged 60 years and younger was established by Mayer et al. 3].

If predictions from personalised mathematical models were reliable and accurate, they could be used for providing better care to AML patients receiving Ara-C consolidation treatment, e.g. in an automatized measurement-decision support loop [4].5. Precisely identifying the period of Ara-C-induced profound leukopenia and modification of treatment schedules based on such predictions might enable prevention of severe infectious complications, sepsis, and thus delay to undergo subsequent treatment cycles. Therefore, by realising timely adherence to consolidation therapy cycles and by avoiding delays in treatment schedule, the density of chemotherapy cycles may be increased and thus deeper remissions and lower relapse rates may be achieved. This may ultimately translate into improved overall survival rates.

Mathematical models for myelosuppression due to various chemotherapy agents have been proposed [6 11] and applied successfully to predict the dynamics of neutrophils $4 \sqrt{12}$. However, this is not the fact for high-dose Ara-C, the most important component in consolidation therapy [2,13]. Pharmacology of Ara-C is particularly difficult, as its exact mechanisms of action both on normal and leukemic cells are not fully understood. The main effect of Ara-C on normal and leukemic proliferating cells is the inclusion of intracellular Ara-C triphosphate (Ara-CTP) into DNA and RNA, which impairs cell replication [14. Yet, the synthesis of intracellular Ara-CTP is saturable such that the clinical success of intermediate-/high-dose Ara-C is not well explained [3, 15, 16. Additional effects are the subject of ongoing research 16, 17.

Here, we surveyed different published and new hypotheses of the pharmacodynamic (PD) effects of Ara-C on WBC dynamics during AML consolidation therapy. We used models for myelosuppression and Ara-C pharmacokinetics (PK) from the literature to quantify prediction accuracies. The general modelling goals were to include possible secondary effects of Ara-C and to obtain a good balance between modelling detail, prediction accuracy, and the number of patient-specific parameters. As a successful methodology, we considered predictions of WBC recovery times (defined as the time when the WBC count recovers above $1 G / L$ ) for different Ara-C schedules and compared them to published average WBC recovery times. 


\section{Methods}

There are many different levels on which hematopoiesis and dynamics of leukemic cells can be modelled [10,18,20]. We analysed models that capture only the most important dynamics for non-leukemic cells and "agglomerate" different physiological effects into simplifying expressions. Leukemic cell dynamics are not considered because of the lack of events (no measurable leukemic cells are present) and because of our focus on WBC recovery. The necessary model extensions to analyse relapse of leukemic cells are also beyond the scope of this paper. All model variations are based on the gold-standard model for myelosuppression developed by Friberg et al. 6] and are tailored to the special case of Ara-C via a parameterised two-compartment PK model. The personalised mathematical models (PMs) were generated by estimating model parameters from clinically measured WBC counts. To get a better understanding of the relation between modelling hypotheses and individual predictions, we used a point estimator approach. We also provide results of a population based estimation for verification and reference.

The procedure is described in detail below. The mathematical approaches to parameter estimation, uncertainty quantification, and statistical analysis, the mathematical equations, the different initial condition strategies, the PK model and the nonlinear mixed-effects modelling approach are described in Appendix S1.

\section{Mathematical Models}

In the interest of providing a solid mathematical basis for future extensions we focused on the most important dynamical features. There are interesting recent extensions and ideas as possible additional effects of Ara-C [16, 17], inclusion of growth factors 1], or modelling also leukemic stem cells 20]. Higher levels of detail come at the price of an increased number of model parameters, which have to be estimated to obtain PMs. The current lack of clinical measurements of leukemic cells or drug and growth factor concentrations leads to identifiability issues with these additional model parameters. Thus we concentrated on agglomerating effects of Ara-C on proliferation and maturation rates.

Fig 1 illustrates the basic assumptions from which we derived twelve model variations of the original Friberg model which we denote by M1-M12 from now on. They differ concerning the number of transition compartments (M1-M3), initial conditions for the differential equations (M3-M5), and model assumptions for the possible effects of Ara-C on proliferation and maturation rates (M5-M12). After intermediate evaluations of accuracies we concentrated on the most promising choice of scaling, transition compartments, and initial conditions, and included different modelling assumptions in the models M6-M12 which are alternatives to M5, our Ara-C extension of the gold-standard model for myelosuppression [6]. Most models refer to previous approaches in the literature and are included for a comprehensive comparison and evaluation of our new hypotheses.

\section{M1-M5: the basic PK/PD model, number of compartments and initial conditions}

In 2002 Friberg et al. published a PK/PD model describing myelosuppression induced by different chemotherapeutic agents (docetaxel, paclitaxel, and etoposide) [6]. The model showed a good trade-off between capturing the important aspects of the dynamics, containing a moderate number of identifiable model parameters, and being applicable for different cytostatic drugs. It has become the gold-standard model in this field with different PK and population-based modifications to topotecan [21], to daunorubicin [22], to a combination therapy of Ara-C (low-dose), etoposide and 


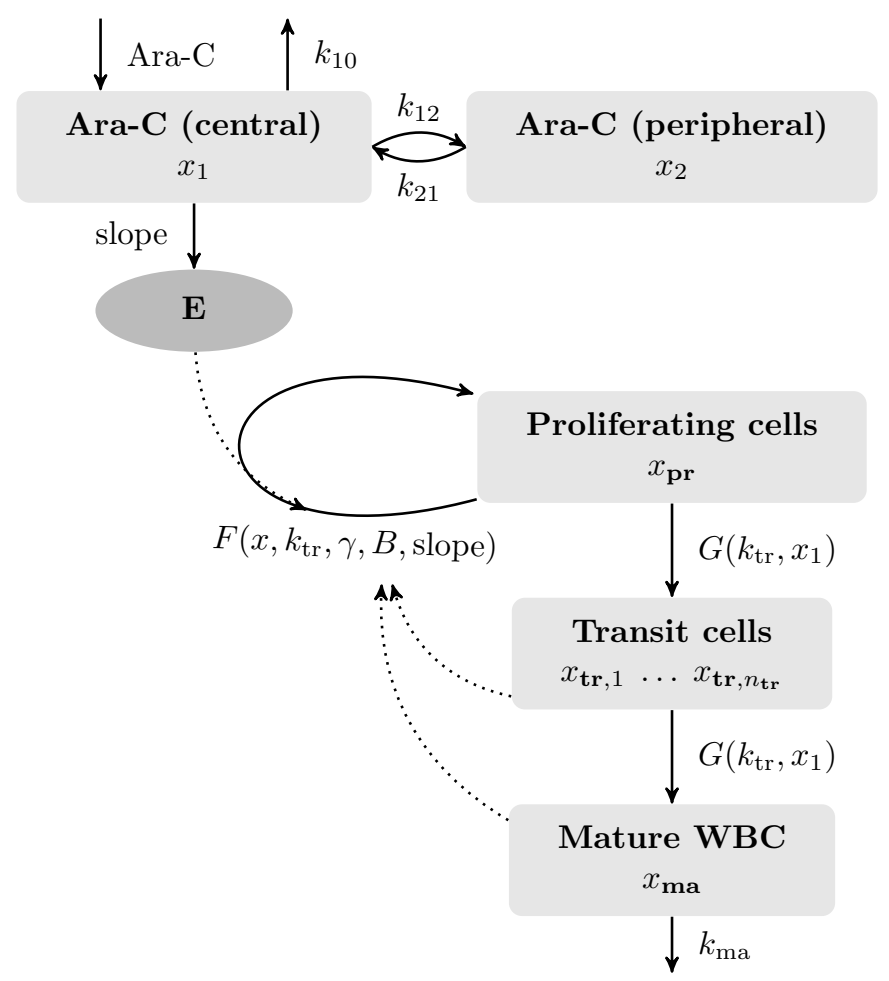

Fig 1. Schematic model from which all mathematical models were derived. We assumed clustering of cells and cytarabine (Ara-C) concentrations in compartments with identical properties. White blood cell (WBC) differentiation is represented by a proliferating compartment $x_{\mathrm{pr}}$, a number $n_{\mathrm{tr}}$ of transit compartments $x_{\mathrm{tr}}$ with different levels of maturation, and a compartment $x_{\text {ma }}$ with mature, circulating WBC. Cells mature with a maturation rate $G$. Mature cells $x_{\text {ma }}$ are dying by the process of apoptosis with a death rate of $k_{\mathrm{ma}}$. The pharmacodynamic effect of Ara-C is described as a $\log$-linear function $E$ targeting the proliferating cells in the bone marrow. It depends on the concentration $x_{1}$ of Ara-C in an assumed central compartment including the circulating blood. The proliferation rate $F$ of $x_{\mathrm{pr}}$ models the replication speed of proliferating progenitor cells. Modelling assumptions were incorporated by choosing different functions $F$ and $G$ (compare Table 1 ). The estimated model parameters used for personalisation were $B$, slope, $k_{\mathrm{tr}}, \gamma$, and initial conditions.

daunorubicin in the induction treatment for AML 8], to a physiologically based PK model for the induction therapy of AML patients with daunorubicin and Ara-C (low-dose) 23, to a combination therapy of carboplatin, etoposide and thiotepa [24], to paclitaxel [10, to an individual-based approach [25], and to drug specific optimisations [9]. The model assumes a clustering of cells in compartments with identical properties. WBC differentiation is represented by a proliferating compartment $x_{\mathrm{pr}}$, a number $n_{\mathrm{tr}}$ of transit compartments $x_{\mathrm{tr}}$ with different levels of maturation, and a compartment $x_{\mathrm{ma}}$ with mature, circulating WBC. Cells mature with a maturation rate constant $G=k_{\mathrm{tr}}$. Mature cells $x_{\mathrm{ma}}$ are dying by the process of apoptosis with a death rate constant $k_{\mathrm{ma}}$. As Monte Carlo simulations were not very sensitive, we fixed $k_{\mathrm{ma}}$ to a constant value as previously proposed 7 .

Apart from different PK models which were linked to the myelosuppression model, also modifications of the structural model were proposed [10.11]. Both models have a 
Table 1. Overview of all investigated mathematical models M1-M12.

\begin{tabular}{lccll}
\hline Model & $\mathbf{n}_{\mathrm{tr}}$ & Initial condition & Proliferation rate $\mathbf{F}\left(\mathbf{x}, \mathbf{k}_{\mathrm{tr}}, \gamma, \mathbf{B}\right.$, slope $)$ & $\mathbf{G}\left(\mathbf{k}_{\mathrm{tr}}, \mathbf{x}_{\mathbf{1}}\right)$ \\
\hline M1 & 6 & $\mathrm{I} 1$ & $(1-E) k_{\mathrm{tr}}\left(B / x_{\mathrm{ma}}\right)^{\gamma}$ & $k_{\mathrm{tr}}$
\end{tabular}

Modelling hypotheses from gold-standard model 6 .

$\begin{array}{lllll}\text { M2 } & 3 & \text { I1 } & (1-E) k_{\mathrm{tr}}\left(B / x_{\mathrm{ma}}\right)^{\gamma} & k_{\mathrm{tr}}\end{array}$

As M1, with $n_{\mathrm{tr}}=3$ transition compartments.

\begin{tabular}{lllll}
\hline M3 & 1 & I1 & $(1-E) k_{\mathrm{tr}}\left(B / x_{\mathrm{ma}}\right)^{\gamma}$ & $k_{\mathrm{tr}}$
\end{tabular}

As M1, with $n_{\mathrm{tr}}=1$ transition compartments.

\begin{tabular}{lllll}
\hline M4 & 1 & I2 & $(1-E) k_{\mathrm{tr}}\left(B / x_{\mathrm{ma}}\right)^{\gamma}$ & $k_{\mathrm{tr}}$
\end{tabular}

As M3, but with more freedom to choose initial values.

$\begin{array}{lllll}\text { M5 } & 1 & \text { I3 } & (1-E) k_{\mathrm{tr}}\left(B / x_{\mathrm{ma}}\right)^{\gamma} & k_{\mathrm{tr}}\end{array}$

As M3, but with more freedom to choose initial values.

\begin{tabular}{lllll}
\hline \hline M6 & 1 & I3 & $k_{\mathrm{tr}}\left(B / x_{\mathrm{ma}}\right)^{\gamma}-E$ & $k_{\mathrm{tr}}$
\end{tabular}

As M5, but assuming a direct killing effect of Ara-C on the proliferating cells.

\begin{tabular}{lllll}
\hline M7 & 1 & I3 & $(1-E) k_{\mathrm{tr}} / S\left(x_{1}\right)\left(B / x_{\mathrm{ma}}\right)^{\gamma}$ & $k_{\mathrm{tr}} / S\left(x_{1}\right)$
\end{tabular}

As M5, but replacing $k_{\text {tr }}$ by $k_{\text {tr }} / S\left(x_{1}\right)$ throughout.

$\begin{array}{lllll}\text { M8 } & 1 & \text { I3 } & (1-E) k_{\mathrm{tr}} / S\left(x_{1}\right)\left(B / x_{\mathrm{ma}}\right)^{\gamma} & k_{\mathrm{tr}}\end{array}$

As M5, but replacing $F$ by $F / S\left(x_{1}\right)$.

$\begin{array}{lllll}\text { M9 } & 1 & \text { I3 } & (1-E) k_{\mathrm{tr}} / S\left(x_{1}\right)\left(B / x_{\mathrm{ma}}\right)^{\gamma S\left(x_{1}\right)} & k_{\mathrm{tr}}\end{array}$

As M8, but also multiplying $\gamma$ with $S\left(x_{1}\right)$.

$\begin{array}{lllll}\text { M10 } & 1 & \text { I3 } & (1-E) k_{\mathrm{tr}}\left(B / x_{\mathrm{ma}}\right)^{\gamma S\left(x_{1}\right)} & k_{\mathrm{tr}}\end{array}$

As M5, but multiplying $\gamma$ with $S\left(x_{1}\right)$, possibly via macrophage activation.

$\begin{array}{lllll}\text { M11 } & 1 & \text { I3 } & (1-E) k_{\mathrm{tr}}\left(B_{\mathrm{bm}} /\left(0.01 * x_{\mathrm{pr}}+0.99 * x_{\mathrm{tr}}\right)\right)^{\gamma} & k_{\mathrm{tr}}\end{array}$

As M5, but feedback depends on bone marrow precursor WBC instead of WBC.

\begin{tabular}{lllll}
\hline M12 & 1 & I3 & $(1-E) k_{\mathrm{tr}}\left(B_{\mathrm{bm}} /\left(0.01 * x_{\mathrm{pr}}+0.99 * x_{\mathrm{tr}}\right)\right)^{\gamma S\left(x_{1}\right)}$ & $k_{\mathrm{tr}}$
\end{tabular}

Combining both modelling assumptions of M10 and M11.

For each mathematical model the number of transition compartments $n_{\mathrm{tr}}$, the initial condition strategy, and the two functions $F$ for proliferation rate and $G$ for maturation rate are specified, compare section Methods and Fig $\mathbf{1}$, respectively. The models M1-M5 have been used mainly to determine the best number of transition compartments and initial condition strategy, which have been kept fixed from M5 onward. Different modelling assumptions are incorporated via different functions $F$ and $G$ in the models M5-M12. An important role has the function $S\left(x_{1}\right):=1+\ln \left(1+c_{V} x_{1}\right)$, compare section Methods. Most important for this paper are the gold-standard model M5 which serves as a reference, and the extended models M10 and M12 as the most promising new candidate models.

more detailed description of the stem cell compartment. The model from Henrich et al. covers a consecutive decrease of the leukocyte's nadir in the treatment cycles achieved by a prior additional compartment mimicking the slow replication of pluripotent stem cells in the bone marrow. Mangas-Sanjuan et al. models a cell-cycle occuring in the bone marrow compartment covering quiescent cells which do not enter the proliferation process and are not sensitive to the pharmacodynamic effect of the treatment.

We used a two-compartment PK model of high-dose Ara-C, which is administered in the consolidation phase, with zero-order input and linear elimination based on published 
drug concentration-time data 26. The corresponding parameters $k_{10}, k_{12}, k_{21}, V_{C}$ were estimated and defined as constants for all further computations. A two-compartment PK model representing a central and peripheral compartment, see Fig $\mathbf{1}$, adequately described the concentration-time data and coincides with the derived values for clearance and the rate constant $k_{10}$ from Table $6[26$. Compared to a published two-compartment PK model for low-dose Ara-C in the induction treatment for AML 8 our clearance and central volume estimates are $40-50 \%$ lower, but within the inter-individual variability (IIV) 8], compare the Appendix S1. Due to the fact that the drug concentration-time data from Figure $2[26$ is not assigned to the patients, the IIV on the clearance and on the central volume from 8 was used for and applied on our PK model to analyse the effect of the PK variability on the different modelling hypotheses in the population approach. A more detailed discussion of the PK model and the comparison with two recently published PK models [8 27] can be found in the Appendix S1. The body surface area BSA and the chemotherapy treatment $u$ /duration were individually fixed by clinical procedure. The PD linking the PK model to the myelosuppression model was modelled by a log-linear function [11

$E=$ slope $\ln \left(1+c_{V} x_{1}\right)$, using the parameter slope for patient-specific calibration and chemotherapeutical effects and the constant $c_{V}$ for unit consistency (see Table S3.

We also implemented a linear PD function with discouraging results. Additionally, we tested a (sigmoid) $E_{\max }$ model without achieving better model accuracies. The function $F\left(x, k_{\mathrm{tr}}, \gamma, B\right.$, slope $)$ is a general description of the proliferation rate of $x_{\mathrm{pr}}$ and incorporates the PD effect $E$ on the proliferating cells, as discussed in Minami et al. 28], Derendorf et al. 29] and applied, e.g. in Quartino et al. 7]. The basic structure of the function $F$ is $(1-E) k_{\mathrm{tr}}\left(B / x_{\mathrm{ma}}\right)^{\gamma}$ in which the mature cells influence the proliferation rate $k_{\mathrm{tr}}$ of $x_{\mathrm{pr}}$ with a feedback term $\left(B / x_{\mathrm{ma}}\right)^{\gamma}$ that leads to higher rates if the number of circulating cells $x_{\mathrm{ma}}$ is below the baseline WBC count $B$, and vice versa. The proliferation exponent $\gamma$ indicates the strength or speed of this feedback. The estimation parameters were $B$, slope, $k_{\mathrm{tr}}$, and $\gamma$ plus a varying approach of initial conditions. We implemented three different strategies to treat initial conditions: I1 assumes a steady state, I2 assumes a steady state only for the proliferating and transient compartments, and I3 penalises deviations from the steady state. I2 and I3 are considered as alternative initial conditions as our clinical data indicate that the steady state assumption after induction phase and between the consolidation cycles may be violated. These two approaches may also account for disease progression effects. A specification of I1, I2, I3 and a discussion of the number of compartments can be found in the Appendix S1. We used I1 and $n_{\mathrm{tr}}=6$ for M1, I1 and $n_{\mathrm{tr}}=3$ for M2, I1 and $n_{\mathrm{tr}}=1$ for M3, I2 and $n_{\mathrm{tr}}=1$ for M4, and I3 and $n_{\mathrm{tr}}=1$ for M5-M12.

\section{M6: Modelling a Direct Killing Effect of Ara-C on the Proliferating Cells}

In the model M6, we chose the proliferation rate as discussed in previous works 9 23, 29] as $F=k_{\mathrm{tr}}\left(B / x_{\mathrm{ma}}\right)^{\gamma}-E$. The main difference to all other models is that the PD effect $E$ is directly multiplied with $x_{\mathrm{pr}}$ and not with $k_{\mathrm{tr}}\left(B / x_{\mathrm{ma}}\right)^{\gamma} x_{\mathrm{pr}}$. Multiplying with $x_{\mathrm{pr}}$ can be seen as a direct (killing) impact of Ara-C on the amount of proliferating cells, whereas the more plausible mechanism-based rationale is the induced reduction of the proliferation rate constant $k_{\mathrm{tr}}$ used in all models except in M6.

\section{M7-M12: Extending the Effects of Ara-C}

The root mean squared error (RMSE) values in Table 2 indicate that model M5 with one transition compartment and initial condition approach I3 provides the highest accuracy after model personalisation compared to M1-M4.

The indirect effect of Ara-C with an impaired proliferation (M5) is more plausible 
Table 2. Root mean squared error (RMSE) values for the models M1-M12.

\begin{tabular}{|c|c|c|c|c|c|c|c|c|c|c|c|c|}
\hline RMSE & M1 & M2 & M3 & M4 & M5 & M6 & M7 & M8 & M9 & M10 & M11 & M12 \\
\hline $\mathrm{Cs}$ & 911 & 0.836 & 0.742 & 0.636 & 0.579 & 0.595 & 0.639 & 0.576 & 0.574 & 574 & .577 & 0.587 \\
\hline 23 Pat & 154 & 011 & .892 & 0.825 & 741 & 0.758 & 785 & 0.753 & 0.738 & .740 & .740 & .741 \\
\hline 14 Pred & $\begin{array}{l}1.209 \\
---2\end{array}$ & 28 & 9 & 1.007 & 0.908 & 0.972 & 0.997 & 0.960 & 0.958 & 0.927 & 0.940 & 0.94 \\
\hline 7 D135 & 1. & 0 & 0 . & 0 . & 0 . & 0.7 & 0.781 & 0 . & 0.7 & 765 & 31 & 88 \\
\hline $5 \mathrm{~d} 135$ & 1.319 & 1.240 & 1.141 & 1.093 & 0.921 & 1.095 & 1.023 & 1.068 & 1.043 & 0.957 & 1.037 & 1.009 \\
\hline$P_{\mathrm{D} 123}$ & 2.404 & 2.218 & 2.241 & 2.258 & 2.011 & 2.014 & 2.324 & 2.006 & 1.996 & 1.996 & 2.029 & 2.022 \\
\hline$P_{\mathrm{D} 12}$ & 1.014 & 0.991 & 1.042 & 0.924 & 0.842 & 0.843 & 1.049 & 0.839 & 0.840 & 0.843 & 0.824 & 0.823 \\
\hline
\end{tabular}

Measured and calculated WBC counts were compared. The estimations and predictions used personalised mathematical models (PMs) that were calculated based on the twelve different mathematical models M1-M12. The first row refers to a personalisation for all 42 consolidation cycles (CCs). The second row shows results for personalisations using all available cycles per patient (Pat). For predictions (Pred) all but one cycle were used for personalisation and the last cycle for cross-validation. Four more rows show the predictions seperated into the different schedules (D135, d135, $P_{\mathrm{D} 123}$ and $\left.P_{\mathrm{D} 12}\right)$. The RMSE values decrease from cycles to patients and from personalisation towards prediction, as expected. Comparing the mathematical models, the accuracy increases with a reduced number of compartments from M1 to M3. The initial condition strategies I2 in M4 and I3 in M5 decrease RMSEs further. M5-M12 all used $n_{\mathrm{tr}}=1$ and I3 and performed equally well, with the slight exception of M7. Note that in particular there is no significant difference between the established gold-standard model M5 and our newly proposed extended model M10.

than a direct killing effect (M6), because Ara-CTP is incorporated into DNA and RNA and impairs cell replication [14]. Therefore, M5 became the reference model for all further analysis. We extended the proliferation rate $F(\cdot)$ and/or the transition rate $G(\cdot)$ in M5 to capture potential secondary effects of Ara-C. To understand the implications of the extensions, we observe that the proliferation rate $F=(1-E) k_{\mathrm{tr}}\left(B / x_{\mathrm{ma}}\right)^{\gamma}$ is negative when $1<E$. This is the case for

$$
c_{V} x_{1}>e^{\text {slope }^{-1}}-1 .
$$

This corresponds to more proliferating cells being in the process of apoptosis than being in the process of cell division. It is important that the feedback term $\left(B / x_{\mathrm{ma}}\right)^{\gamma}$ increases the absolute value of $F$ for $B>x_{\mathrm{ma}}$, and decreases it for $B<x_{\mathrm{ma}}$. Therefore, an analysis of $F$ always has to consider all four cases related to the signs of $1-E$ and of $B-x_{\mathrm{ma}}$. Inspired by the log-linear behaviour of the PD effect $E$, we chose

$$
S\left(x_{1}\right):=1+\ln \left(1+c_{V} x_{1}\right) .
$$

This monotonously increasing function is applied to different expressions in M5.

In M7 we replaced the transition rate $k_{\mathrm{tr}}$ by $k_{\mathrm{tr}} / S\left(x_{1}\right)$ throughout M5. This results in an Ara-C induced reduction of the transition rate.

In M8 we replaced the complete feedback function $F$ in M5 by $F / S\left(x_{1}\right)$. This models an Ara-C induced decreased auto-feedback of the proliferating cells. For high values of $x_{1}$, i.e. when (1) holds, this results in a decreased killing of proliferative cells. For values $x_{1}>0$ below that boundary, we get a decreased positive proliferation rate.

In M9 we replaced both the complete feedback function $F$ by $F / S\left(x_{1}\right)$ and the proliferation exponent $\gamma$ by $\gamma S\left(x_{1}\right)$. Again, depending on $x_{1}$ either the killing or the proliferation rate of $x_{\mathrm{pr}}$ are decreased by $F / S\left(x_{1}\right)$. In addition, the impact depends on 
whether the WBC count is below or above the baseline: for $x_{\text {ma }}<B$ we have an increased killing/proliferation rate $\left(B / x_{\mathrm{ma}}\right)^{\gamma S\left(x_{1}\right)}>\left(B / x_{\mathrm{ma}}\right)^{\gamma}$ and vice versa.

In M10 we replaced the proliferation exponent $\gamma$ in M5 by $\gamma S\left(x_{1}\right)$. This is motivated by the observation that the feedback term with exponent $\gamma$ is related to the endogenous G-CSF [6], and the assumption that higher concentrations of Ara-C coincide with activation of macrophages and hence increased G-CSF secretion. In contrast to M9 the function $F$ itself is not scaled. Like in M9, the $\gamma S\left(x_{1}\right)$ scaling results in an increase of killing/proliferation rates for WBC counts below the baseline, and a decrease else.

In M11 we replaced the quotient $B / x_{\text {ma }}$ by a comparison between cells in the bone marrow and their baseline value. We estimated that about $1 \%$ of the WBC precursor cells in the bone marrow are in the proliferating compartment $x_{\mathrm{pr}}$, and $99 \%$ in the transition compartment $x_{\mathrm{tr}}$.

In M12 we combined the extensions from M10 and M11.

\section{Clinical Data (high density WBC counts) \& Personalisation}

AML patients who had received induction therapy (commonly defined as anthracyclineand Ara-C-based 7+3 regimen [2]) resulting in complete remission and who did not receive granulocyte-colony stimulating factors (G-CSF) during the post-remission consolidation therapy were eligible for data analysis. We focused on patients who did not receive growth factor support, as such effects were not yet accounted for in our mathematical models. Almost daily WBC counts from 42 consolidation Ara-C cycles (CCs) of 23 AML patients (median 62 years, 14 male, mostly de novo AML (19/23), mostly AML FAB-M2 (9/19), mostly intermediate cytogenetic risk (12/20)) from 2008 to 2015 were analysed from clinical charts provided by the Department of Hematology and Oncology, Magdeburg University Hospital, Magdeburg, Germany. The data were retrospectively collected and pseudonymised from records of the clinical routine.

Interventions were not performed for this work. All clinical procedures were performed in accordance with the general ethical principles outlined in the Declaration of Helsinki. For this reason no patients' agreements were required. The CCs were partitioned in one, two, and three consecutive CCs from nine, nine, and five patients, respectively. Four different schedules D135, d135, D123, or D12, in which the numbers correspond to treatment days $1,2,3$, and 5 , respectively, d to intermediate-dose Ara-C (i.e. $1 \mathrm{~g} / \mathrm{m}^{2}$ per body surface area (BSA) twice a day over three hours) and D to high-dose Ara-C (i.e. $3 \mathrm{~g} / \mathrm{m}^{2}$ twice a day), were administered 24,14 , two, and two times. Patient $P_{\mathrm{D} 123}$ (62 years, male) received two cycles of D123. Patient $P_{\mathrm{D} 12}$ (64 years, female) received two cycles of D12. The 21 other patients received 1-3 D135 cycles (median 57 years, 8 male, 4 female) or d135 cycles (median 68 years, 5 male, 4 female).

We used all 42 CCs to personalise our mathematical models M1-M12 performing point estimations (individual approach) and M3, M10 (with I1), the model from Henrich et al. [10] and the model from Mangas-Sanjuan et al. [11 applying nonlinear mixed-effects modelling (population approach).

The ordinary differential equations comprise states (drug concentrations and cell counts), control (chemotherapy schedule), and model parameters. Model parameters are variable numbers and the main tool for personalisation. In the interest of identifiability we calculated values for PK parameters a priori from published data [26] and fixed others to values from the literature. We then used individual and population parameter estimations to obtain the remaining model parameters $B$, slope, $k_{\mathrm{tr}}$, and $\gamma$ plus a varying initial condition approach such that measured WBC counts from one or several cycles were fitted in an optimal way. Once the model parameters have particular values, the model is called PM. A PM can be solved numerically, resulting in calculated cell counts at arbitrary time points that can be used for further analysis. 


\section{Prediction \& Cross-Validation}

The PMs were then used to predict (simulate) and cross-validate WBC counts for the last CC of 14 patients for whom at least two consecutive CCs are available. Additionally, we calculated predicted $t_{\text {rec }}$ values from our 42 PMs (see Clinical Data \& Personalisation) applying D123 and D135 schedules and compared the descriptive statistics with published average $t_{\text {rec }}$ values from a subset of data (367 CCs of 208 AML patients, no G-CSF support) of the AMLSG 07-04 trial in which the schedules D123 and D135 after $7+3$ regimen were analysed [30]. The published AMLSG 07-04 30] trial does not provide WBC counts to obtain new PMs, therefore we used the median of observed $t_{\text {rec }}$ values for D123 and D135 Ara-C schedules. In the interest of a fair comparison (i.e., to avoid comparison with the value 0) we excluded five out of $42 \mathrm{PMs}$ for which at least one prediction (M1-M12 with either D123 or D135) resulted in no WBC counts below the threshold value. Further, we predicted $t_{\text {rec }}$ values for two Ara-C schedules in which a constant administration of Ara-C throughout days 1-5, with either $100 \mathrm{mg} / \mathrm{m}^{2}$ or $400 \mathrm{mg} / \mathrm{m}^{2}$ was given. These schedules, together with D135, have been clinically analysed for 1088 AML patients (median 52, 568 male) by Mayer et al. [3], and the superiority of D135 with respect to disease-free survival rates and remaining in continuous complete remission after four years has been shown but no $t_{r e c}$ values were reported. Finally, we analysed the effect of the inter-individual PK variability on the $t_{r e c}$ values derived by the models M3 and M10 (with I1). We applied schedules D123 and D135 with fixed population parameter values for $B$, slope, $k_{\mathrm{tr}}$, and $\gamma$ and performed 500 simulations each with randomly chosen values from the inter-individual variability (IIV) for the PK parameters clearance and central volume.

All experiments were performed to analyse the 12 proposed models with respect to WBC count and $t_{\text {rec }}$ predictability.

\section{Schedule Timing}

After verifying the predictability performance of the PMs, we performed a simulation study in which we demonstrated a further possible application of the PMs in clinical practice. We analysed the impact of the treatment timing on the individual nadir values. For each of the 14 patients, for whom at least two consecutive CCs were available, the nadir of the last CC was compared to 20 simulated nadirs. These nadirs resulted from simulations using the patient's PMs (second row of Table 2 in which the timing of the last $\mathrm{CC}$ was varied daily with the maximal starting variation of 10 days earlier or later.

\section{Results}

\section{Accuracy of PMs with fixed Ara-C schedule}

Table 2 shows statistics for PMs derived from M1-M12, for a pure estimation (using all available WBC counts to personalise the model) and for a cross-validation (using all but the last CC for personalisation). The accuracies depend strongly on the number of compartments and initial condition strategy (M1-M5), but are stable against modelling uncertainty with respect to possible effects of Ara-C (RMSE values for prediction between 0.997 and 0.908 for M5-M12). These values were even better when the standard schedule D135 was applied in the estimated and predicted cycles.

Goodness-of-fit plots in Fig $\mathbf{2} \mathbf{a}-\mathbf{b}$ and Fig $\mathbf{S 1}$ visualise the good match between model predictions and measured WBC counts (respectively observed $t_{r e c}$ values) around the nadir and a wider spread of large WBC counts. Fig $\mathbf{2} \mathbf{c}-\mathbf{d}$ indicate the involved uncertainty derived from the individual variance-covariance matrix by means of Monte 
Carlo simulations (for more information see Appendix S1). The uncertainty reduces when more WBC counts are used, and the accuracy increases.

The WBC counts around the nadir are explained well by all models for fixed Ara-C schedules (either D135 or D123), as shown in Fig $\mathbf{3} \mathbf{a}-\mathbf{d}$ for two exemplary patients and in Fig $\mathbf{S 2}$ S3 for the other 12 patients with at least two consecutive CCs.

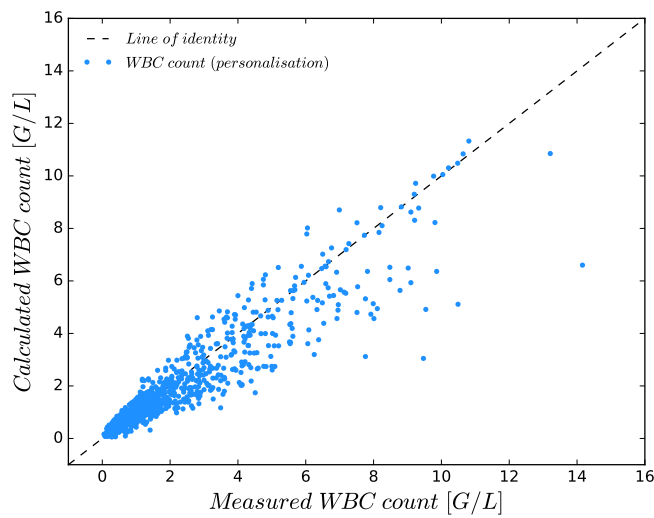

(a) Goodness-of-fit plot for M10. Shown are measured versus calculated white blood cell (WBC) counts. Models were personalised using complete data sets of one to three cycles from 23 patients. The measured counts around the nadir coincide well $(\mathrm{RMSE}=0.740)$ with the calculated WBC counts.

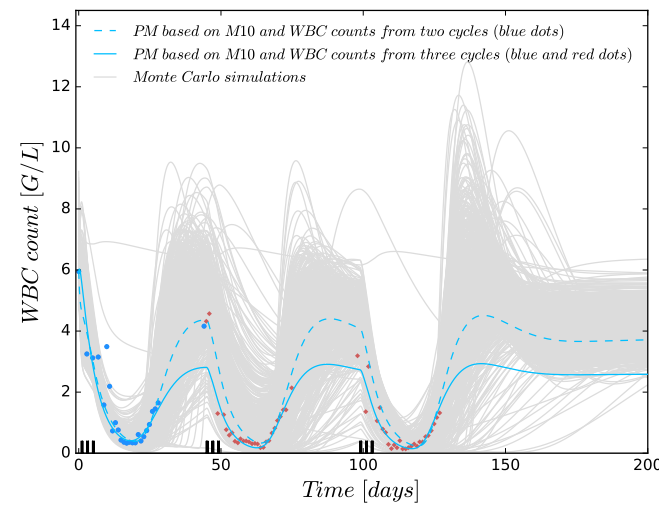

(c) PMs based on M10 and either personalisation with WBC counts from one or from all three cycles. 1000 Monte Carlo simulations after personalisation with WBC counts from one cycle were used to indicate the propagated probability density function. We ensured that all samples are within the $1 \%$ to $99 \%$ quantile such that also some outliers are observable.

Fig 2. Visualisation of predictive accuracies of personalised mathematical models (PM).

\section{Accuracy of PMs with altered Ara-C schedule}

Fig 3 e-f show two cases where D135 is used for personalisation and D123 for prediction (and vice versa). Here, M9, M10, and M12 have a faster (slower) cross-validated WBC counts from the last (b) As (a), but cross-validated: WBC counts from the last cycle of patients were not used for personalisation, but compared to predictions $(\mathrm{RMSE}=0.927)$. The plot shows cycle in red, others in blue. The plots are prototypical for M1-M12.

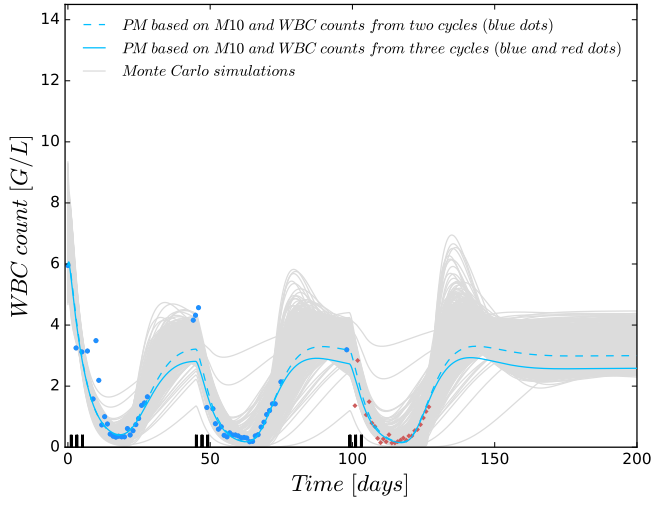

(d) As (c), but using WBC counts from the first two cycles for personalisation. More measurements lead to higher prediction accuracy. The uncertainty tube tightens and the predicted trajectory gets closer to the solution that used all available WBC counts. 
hematological recovery for D123 (D135). All three models assume that the proliferation speed $\gamma$ depends on the Ara-C concentration. This modelling assumption is visualised in a different way for M5, M10 and M12 in Fig $\mathbf{S 4}-\mathbf{S 6}$. It is shown that the proliferation rate $F$ of M10 and M12 compared to the proliferation rate of M5 behaves in such a way that a faster WBC recovery for D123 schedules is achieved. This indicates that the comparison of WBC recovery times between D123 and D135 treatments is a suitable criterium for model discrimination. 


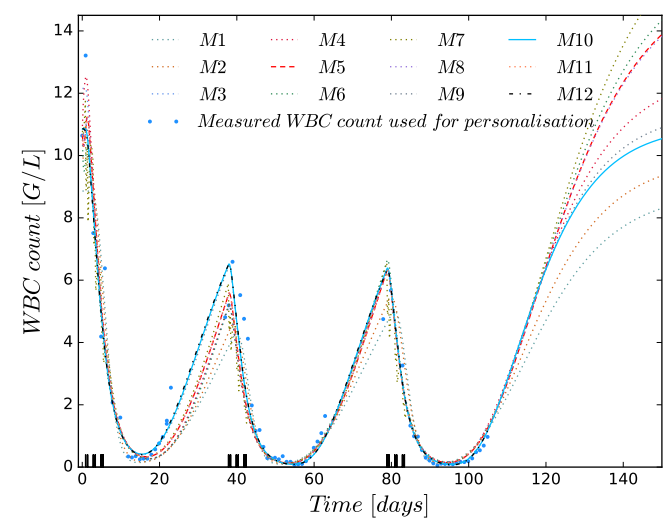

(a) Reproducability: all 12 PMs based on M1-M12 are able to explain the measured WBC counts.

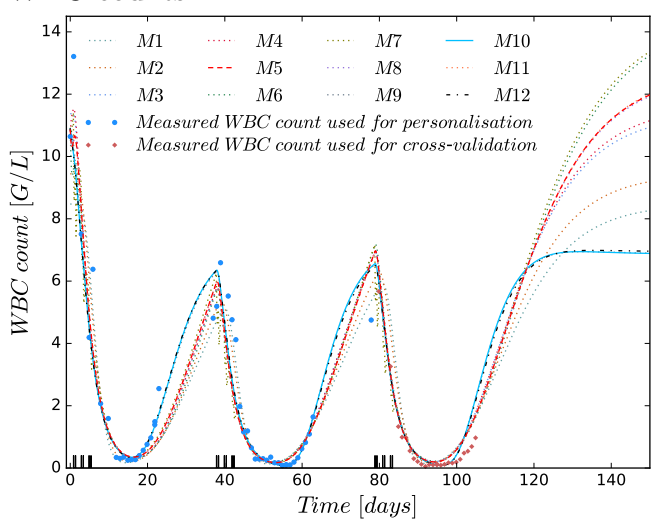

(c) Cross-validated prediction: all PMs explain the WBC counts well, also in the predicted third cycle.

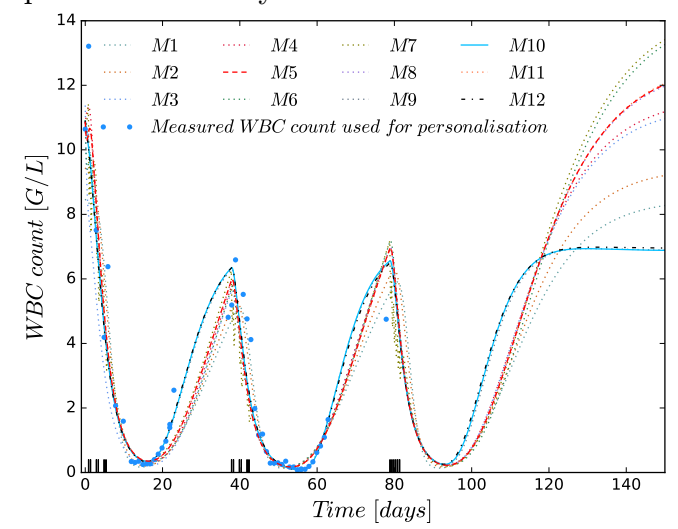

(e) Varied Ara-C schedule: prediction of D123 in the third cycle for a PM based on two D135 cycles shows faster WBC recovery for M9, M10, and M12.

Fig 3. Comparison of personalised models (PMs) based on M1-M12 and white blood cell (WBC) data. Patient with three D135 cycles (left) and patient $P_{\mathrm{D} 123}$ with two D123 cycles (right), as indicated on the x-axis. The PMs exemplify reproducability (first row), predictability (second row) and simulation of a different schedule in prediction than estimation (third row).

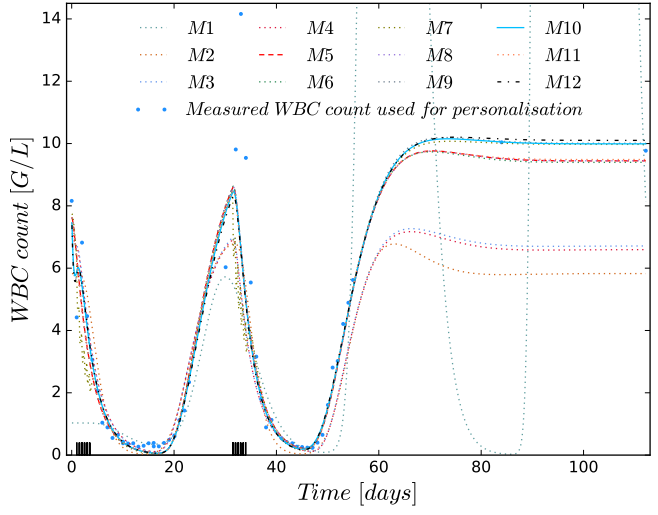

(b) As in (a), all PMs explain the measured WBC counts well, particularly around the nadirs.

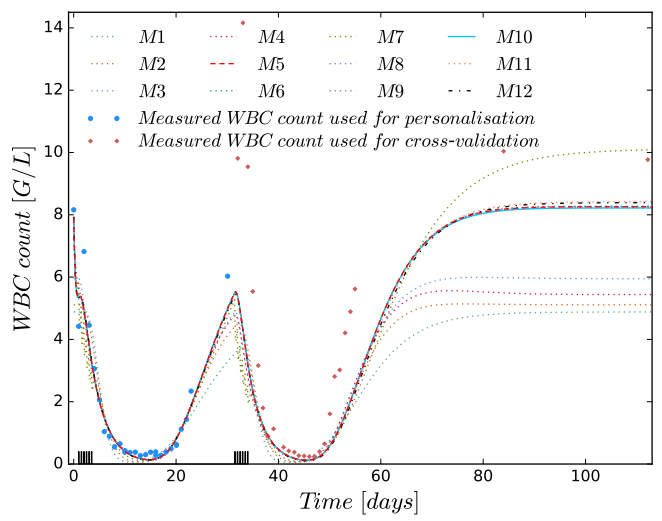

(d) As in (c), here with a slightly too slow predicted recovery time in the second cycle for all models.

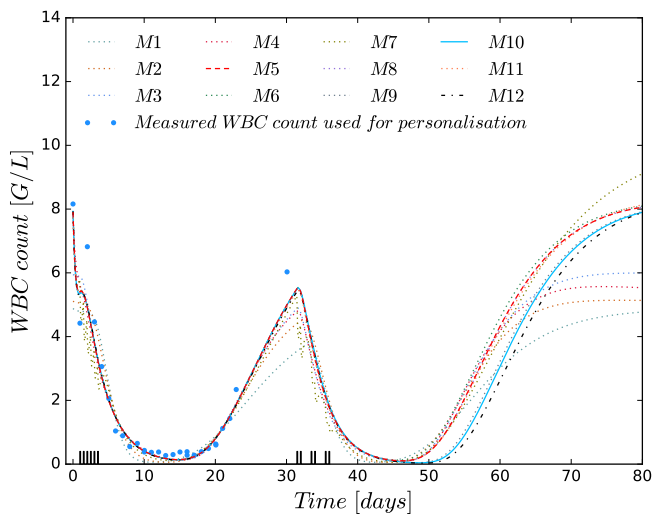

(f) Prediction of D135 in the second cycle for a PM based on one D123 cycle shows slower WBC recovery times for M9, M10, and M12. 
Table 3 shows results for a comparison of predicted and measured $t_{\mathrm{rec}}$ values. We used 444 PMs (using M1-M12 and clinical data from 37 cycles with schedules D135, d135, D123 and D12 from section Clinical Data \& Personalisation) to predict the outcome of D135 and D123 schedules. The median values of the predicted $t_{\text {rec }}$ were compared to the values from a subset of data (108 with D135 and 259 with D123 schedules) from the AMLSG 07-04 trial [30]. M9, M10, and M12 resulted in roughly 4 days faster $t_{\text {rec }}$ for D123 compared to D135, similar to the clinical result from the literature and in contrast to the 1 day difference of M5. The individual results have been qualitatively confirmed by the predicted $t_{\mathrm{rec}}$ values from the population approach (see Table S4). The models from Henrich et al. and Mangas-Sanjuan et al. were not further considered, as both models simplified to the original Friberg model after parameter estimation. For the model from Henrich et al., the estimated population parameter value $f_{t r}$ was 0.96 , supporting the visual assessment that the patients' nadirs are not decreasing during the CCs. The estimated parameter values $k_{\text {cycle }}=0.0009$ and $F_{\text {prol }}=0.941$ of the model from Mangas-Sanjuan et al. yielded a non-existing stem cell cycle. A possible reason for the non-identifiability of the parameters might be the limited schedule variation. The authors state that a vast variation of schedules has to be available for parameter identification [11].

The simulation study analysing the effect of the PK variability on the resulting recovery times of schedules D123 and D135 for models M3 and M10 (with I1) revealed that model M10 was more sensitive to different high-dose Ara-C treatment schedules compared to model M3 despite the high inter-individual PK variability. This was verified in Fig [S9 presenting boxplots of 500 simulated $t_{r e c}$ values for both models and schedules with IIV on the PK.

\section{Schedule Timing}

We analysed the impact of different treatment starts of the last CCs with respect to subsequent nadir values. A comparison to the clinically observed nadir values indicated a large potential for clinical improvement, i.e., a higher nadir value due to a different treatment timing (see Fig 4(a)). Fig 4(b) exemplarily shows the WBC dynamics for different treatment timings. Earlier (later) starts resulted in sequentially higher (lower) nadir values.

\section{Discussion}

High-density WBC counts from 23 AML patients were collected and used to personalise 12 mathematical models and analyse their prediction accuracy with respect to different modelling hypotheses and treatment schedules. The high prediction accuracies of the PMs, especially around the nadir, confirm previous claims 4, 12, that the general approach of in-silico studies can be used for clinical decision support, e.g. to monitor and predict WBC dynamics.

In combination with clinical expertise on the impact of schedules on relapse probabilities, this might have an important clinical impact via altered treatment schedules which might eventually result in improved survival rates. To evaluate or even optimise the dosage of Ara-C, PMs may not be appropriate, though. We showed that an analysis based on a fixed chemotherapy schedule can not discriminate between different modelling hypotheses. The agglomerative nature of the mathematical models leads to a choice of model parameters that is not only personalised to the patient, but also to the applied schedule. Therefore, we used, different schedules for personalisation and prediction to overcome this problem and to allow discrimination of the models. This approach facilitated to distinguish between the modelling hypotheses implemented 
Table 3. Double cross-validation with clinical data from two independent clinical trials.

\begin{tabular}{|c|c|c|c|c|c|c|c|c|c|c|c|c|c|c|c|}
\hline & & \multicolumn{4}{|c|}{$\mathbf{t}_{\mathrm{rec}}$} & \multicolumn{2}{|c|}{$\mathrm{t}_{\mathrm{rec}}^{\mathrm{D} 135-\mathrm{D} 123}$} & \multicolumn{4}{|c|}{$\mathbf{t}_{\text {leu }}$} & \multicolumn{4}{|c|}{ nadir } \\
\hline & & \multicolumn{2}{|c|}{ D123 } & \multicolumn{2}{|c|}{ D135 } & & & \multicolumn{2}{|c|}{ D123 } & \multicolumn{2}{|c|}{ D135 } & \multicolumn{2}{|c|}{ D123 } & \multicolumn{2}{|c|}{ D135 } \\
\hline \multirow{2}{*}{\multicolumn{2}{|c|}{$\begin{array}{l}\text { Clinical Data } \\
{ }_{n} D 123=2,{ }_{n}{ }^{D 135}=24\end{array}$}} & 19.3 & \pm 1.0 & & \pm 2.9 & - & - & 11.5 & \pm 3.5 & 12.5 & \pm 4.7 & 0.3 & \pm 0.0 & & \pm 0.2 \\
\hline & & (18.6 & 20.1) & (14.1 & 7.1) & - & - & $(9.1$ & $14.0)$ & $(5.0$ & $20.0)$ & $(0.24$ & $0.27)$ & $(0.1$ & $0.8)$ \\
\hline \multirow{2}{*}{\multicolumn{2}{|c|}{$\begin{array}{l}\text { Subdata (AMLSG 07-04) } \\
{ }_{n}{ }^{D 123}=259,{ }_{n}{ }^{D 135}=108\end{array}$}} & 18.0 & - & 22.0 & - & - & - & - & - & - & - & - & - & - & - \\
\hline & & $(-$ & $-)$ & $(-$ & $-)$ & - & - & - & - & - & - & - & - & - & ـ \\
\hline \multicolumn{2}{|c|}{ Model Group } & \multicolumn{2}{|c|}{ D123 } & \multicolumn{2}{|c|}{ D135 } & & & \multicolumn{2}{|c|}{ D123 } & \multicolumn{2}{|c|}{ D135 } & \multicolumn{2}{|c|}{ D123 } & \multicolumn{2}{|c|}{ D135 } \\
\hline \multirow{2}{*}{ M1 } & \multirow{2}{*}{$37 \mathrm{PMs}$} & 21.9 & \pm 3.7 & 22.6 & \pm 3.7 & 0.7 & \pm 0.2 & 13.3 & \pm 4.5 & 13.3 & \pm 4.6 & 0.2 & \pm 0.2 & 0.2 & \pm 0.2 \\
\hline & & $(16.0$ & $34.8)$ & $(17.0$ & $35.6)$ & $(0.3$ & 1.0) & $(7.5$ & $28.3)$ & $(7.5$ & $28.5)$ & $(0.0$ & $0.6)$ & $(0.0$ & $0.6)$ \\
\hline \multirow{2}{*}{ M2 } & & 22.2 & \pm 3.5 & 22.6 & \pm 3.5 & 0.5 & 0.2 & 13.3 & \pm 4.2 & 13.4 & \pm 4.2 & 0.3 & \pm 0.2 & 0.3 & \pm 0.2 \\
\hline & & $(16.4$ & $33.3)$ & $(17.4$ & $33.8)$ & $(0.2$ & 1.0) & $(7.5$ & $26.2)$ & $(7.6$ & 26.4) & $(0.1$ & $0.6)$ & $(0.1$ & $0.6)$ \\
\hline & $37 \mathrm{PMs}$ & 22.3 & \pm 2.7 & 23.2 & \pm 2.7 & 0.9 & 0.1 & 13.2 & \pm 3.7 & 14.1 & \pm 3.6 & 0.4 & \pm 0.2 & 0.4 & \pm 0.2 \\
\hline & & $(17.1$ & 30.7) & $(18.2$ & $31.7)$ & $(0.8$ & 1.3) & $(7.2$ & 23.3) & $(8.3$ & 23.9) & $(0.1$ & $0.8)$ & $(0.1$ & $0.8)$ \\
\hline & & 22.4 & \pm 2.8 & 23.3 & \pm 2.7 & 0.9 & 0.1 & 13.2 & \pm 3.7 & 14.1 & \pm 3.6 & 0.4 & \pm 0.2 & 0.4 & \pm 0.2 \\
\hline & & $(17.0$ & 30.9) & $(18.1$ & 31.9) & $(0.8$ & 1.2) & $(8.5$ & 23.5) & $(9.4$ & 24.1) & $(0.1$ & $0.8)$ & $(0.1$ & $0.7)$ \\
\hline & & 22.4 & \pm 3.1 & 23.3 & \pm 3.0 & 0.9 & 0.2 & 12.7 & \pm 4.0 & 13.5 & \pm 4.0 & 0.3 & \pm 0.2 & 0.4 & \pm 0.2 \\
\hline & & $(16.8$ & $32.5)$ & $(18.0$ & $33.5)$ & $(0.1$ & 1.2) & (6.6 & 25.5) & $(7.6$ & 26.1) & .1 & $0.9)$ & $(0.1$ & 0.9) \\
\hline there & 24 & 22.6 & \pm 2.5 & 23.4 & \pm 2.4 & 0.9 & 0.2 & 14.9 & \pm 3.5 & 15.4 & \pm 3.5 & 0.3 & \pm 0.2 & 0.3 & \pm 0.2 \\
\hline & 135 PMs & (16.8 & 27.1) & $(18.0$ & 27.2) & $(0.1$ & 1.2) & $(9.9$ & 20.1) & $(10.7$ & 20.6) & (0.1 & $0.7)$ & (0.1 & $0.7)$ \\
\hline an & & 22.5 & \pm 3.4 & 23.4 & \pm 3.4 & 0.9 & 0.1 & 11.8 & \pm 4.5 & 12.7 & \pm 4.4 & 0.2 & \pm 0.2 & 0.2 & \pm 0.1 \\
\hline & $\mathrm{d}$ & $(18.8$ & $32.5)$ & $(19.7$ & $33.5)$ & $(0.6$ & 1.1) & $(7.2$ & $25.5)$ & $(8.0$ & 26.1) & (0.1 & $0.6)$ & $(0.1$ & $0.6)$ \\
\hline & & 22.6 & \pm 3.4 & 22.8 & \pm 3.3 & 0.2 & 0.2 & 13.2 & \pm 4.0 & 13.2 & \pm 4.0 & 0.4 & \pm 0.2 & 0.4 & \pm 0.2 \\
\hline & & $(14.5$ & $32.0)$ & $(15.3$ & 32.4) & $(0.0$ & $0.8)$ & $(4.6$ & $25.0)$ & $(4.7$ & 24.9) & $(0.1$ & $0.9)$ & $(0.1$ & $0.9)$ \\
\hline & & 22.5 & \pm 2.7 & 23.0 & \pm 2.7 & 0.5 & 0.2 & 12.8 & \pm 4.3 & 13.2 & \pm 4.7 & 0.3 & \pm 0.4 & 0.3 & \pm 0.4 \\
\hline & & $(17.8$ & 31.0) & $(18.4$ & $31.8)$ & $(0.0$ & 1.1) & $(0.0$ & 23.6) & $(0.0$ & 23.9) & $(0.1$ & $2.7)$ & $(0.0$ & $0.9)$ \\
\hline & & 21.7 & \pm 2.7 & 22.6 & \pm 2.6 & 0.9 & 0.1 & 12.6 & \pm 4.0 & 13.5 & \pm 3.9 & 0.3 & \pm 0.2 & 0.4 & \pm 0.2 \\
\hline & & $(16.7$ & 29.4) & $(17.9$ & $30.5)$ & $(0.6$ & 1.3) & $(6.5$ & 22.1) & $(7.3$ & 22.7) & 0.1 & $0.8)$ & $(0.1$ & $0.7)$ \\
\hline & & 20.1 & \pm 2.7 & 23.7 & \pm 2.6 & 3.1 & 0.9 & 10.5 & \pm 4.0 & 14.7 & \pm 3.6 & 0.4 & \pm 0.2 & 0.3 & \pm 0.2 \\
\hline & & $(16.0$ & 28.9) & $(18.6$ & $31.6)$ & $(0.8$ & $5.2)$ & $(3.4$ & 21.7) & $(7.3$ & 23.9) & $(0.1$ & $0.9)$ & $(0.0$ & $0.7)$ \\
\hline & & 20.3 & \pm 3.2 & 24.2 & \pm 3.2 & 3.5 & 1.0 & 11.6 & \pm 4.4 & 15.2 & \pm 3.9 & 0.4 & \pm 0.2 & 0.3 & \pm 0.2 \\
\hline & & (15.4 & $32.4)$ & $(18.9$ & $35.7)$ & ( 0.9 & 5.8) & (1.6 & 25.5) & $(7.6$ & 28.4) & (0.1 & 1.0) & $(0.0$ & $0.7)$ \\
\hline there & 24 & 20.4 & \pm 2.3 & 23.7 & \pm 2.2 & 3.1 & 0.6 & 12.8 & \pm 3.9 & 15.4 & \pm 3.3 & 0.4 & \pm 0.2 & 0.3 & \pm 0.2 \\
\hline & D135 PMs & (16.0 & 23.7) & (18.9 & 26.7) & $(2.2$ & 4.8) & (6.1 & 18.3) & $(10.5$ & 20.5) & $(0.2$ & $0.9)$ & (0.1 & $0.7)$ \\
\hline anc & & 21.7 & \pm 4.3 & 26.2 & \pm 4.0 & 4.4 & 1.2 & 10.5 & \pm 5.4 & 15.2 & \pm 4.9 & 0.4 & \pm 0.2 & 0.1 & \pm 0.2 \\
\hline & d135 & (15.4 & 32.4) & (19.9 & $35.7)$ & ( 0.9 & 5.8) & (1.6 & 25.5) & (7.6 & 28.4) & (0.1 & 1.0) & (0.1 & $0.6)$ \\
\hline & & 22.3 & \pm 3.0 & 23.2 & \pm 2.9 & 0.9 & 0.1 & 12.7 & \pm 4.1 & 13.5 & \pm 4.0 & 0.3 & \pm 0.2 & 0.4 & \pm 0.2 \\
\hline & 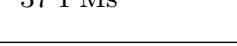 & $(16.7$ & 32.6) & $(17.9$ & 33.7) & $(0.7$ & 1.2) & $(6.5$ & 25.6) & $(7.6$ & 26.2) & $(0.1$ & $0.8)$ & $(0.1$ & $0.7)$ \\
\hline & & 20.4 & \pm 3.3 & 24.0 & \pm 3.4 & 4.0 & 1.2 & 11.9 & \pm 4.4 & 15.4 & \pm 3.9 & 0.4 & \pm 0.2 & 0.2 & \pm 0.2 \\
\hline & & (15.6 & $32.9)$ & (18.7 & $36.4)$ & $(2.0$ & $8.2)$ & (3.1 & $26.0)$ & (8.4 & 29.1) & (0.1 & $0.9)$ & (0.0 & $0.6)$ \\
\hline
\end{tabular}

Shown are the median, standard deviation, minimum and maximum (in brackets) of $t_{\mathrm{rec}}$, the leukopenia time $t_{\text {leu }}$ (the number of days with WBC count $\leq 1 \mathrm{G} / \mathrm{L}$ ) and nadir for D123 and D135 schedules. The first two rows show values from two independent clinical studies that serve as a comparison. The second part of the table shows prediction results. Predictions were calculated with PMs from our clinical data with underlying mathematical models M1-M12. Model M5 explained well the outcome of schedule D135, but showed a significant mismatch of more than three days for schedule D123. The predictions using the extended model M10 were better for schedule D123. See also Fig $\mathbf{3}$ e-f for an illustrated comparison between M5 and M10. Model M9 and M12 were also promising, but we focused on M10 applying Ockam's razor.

in models M5-M12 and enabled to find the suitable model assumption considered in M9, M10, and M12. In our opinion this procedure should be routinely applied, preferably using high density WBC counts for different schedules in the same patients. As an alternative to such a tedious clinical study we suggest to use average $t_{\text {rec }}$ values as a discrimination criterion for competing models. 


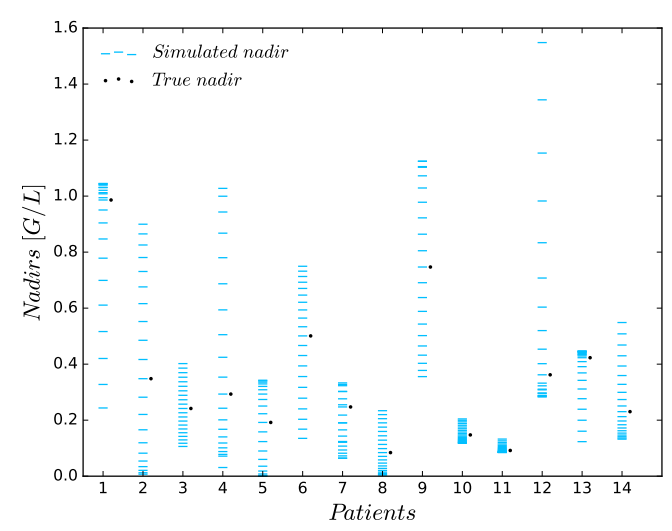

(a)

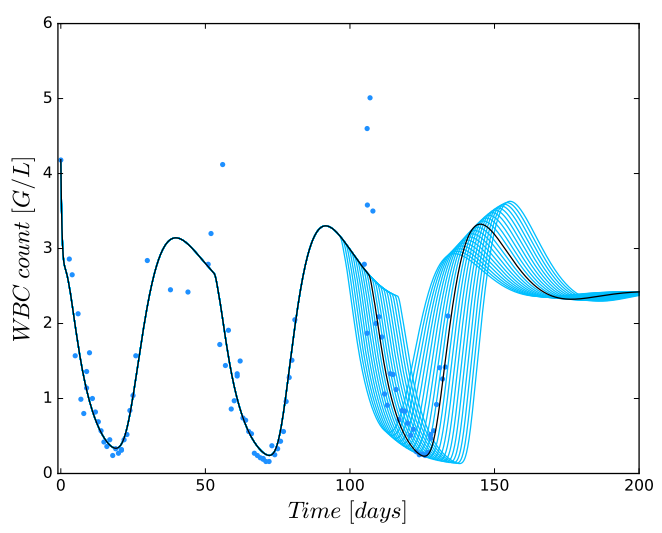

(b)

Fig 4. Analysing the influence of treatment timing on nadir values.

(a) Simulation study in which 20 simulated nadirs were compared with the true nadir of the last $\mathrm{CC}$ for the 14 patients who have more than one CC. The simulated nadirs were computed by using the patient's PM (second row of Table 2) and varying the start of the last CC daily with the maximal starting variation of 10 days earlier or later. (b) Exemplary variation of the CC start for one patient. An earlier (later) start results in a larger (lower) nadir.

Comparing our clinical data and the AMLSG 07-04 trial with respect to $t_{r e c}$ in Table 3 and Table $\mathbf{S 4}$, our observed WBC recovery times are for both schedules 1 day longer. This can be explained by the age difference between patients in our clinical data (median 62 and 57 years for D123 and D135, respectively) and the subdata of the AMLSG 07-04 trial (median of all patients in the trial 49 years) and a related statistical analysis: Jaramillo et al. 30 found in a multivariable analysis a significantly longer WBC recovery for older patients (hazard ratio of a 10-year age difference, $0.89 ; \mathrm{P}=$ $0.001) 30$ and a significantly shorter WBC recovery for patients receiving D123 compared to the reference group D135 (hazard ratio, 1.94; $\mathrm{P}<0.0001$ ) 30] which coincides well with our findings. The comparison of $t_{\mathrm{rec}}$ values for D123 and D135 treatments indicates that models M9, M10, M12 are the best candidates among M1-M12 for future work on the simulation and optimisation of intermediate to high-dose Ara-C treatment schedules.

Summarising, we extended the gold-standard model for myelosuppression $[6]$ to the most important component in consolidation therapy 2, 13, Ara-C, and showed that one modelling assumption was important for a faster WBC count recovery for D123 schedules. In models M9, M10, and M12 we assumed that the Ara-C concentration has a direct impact on the proliferation speed. As stated above, such a modelling assumption has an agglomerative nature and the underlying physiological processes are still unknown. We speculate that a high Ara-C concentration might lead to an increased number of cell deaths and thereby induces phagocytosis and macrophage activation, which in turn might increase G-CSF secretion and hence proliferation speed. Future G-CSF concentration measurements for AML patients during consolidation cycles of D123 and D135 treatments and a comparison of our extended models with Quartino's 31] integrated G-CSF-myelosuppression model may shed light on this speculation.

\section{Conflict of interest}

The authors declare no competing financial interests. 


\section{Funding}

This project has received funding from the European Research Council (ERC) under the European Union's Horizon 2020 research and innovation programme (grant agreement No 647573) and from the "International Max Planck Research School (IMPRS) for Advanced Methods in Process and System Engineering" in Magdeburg.

370

371

372

373

\section{Acknowledgements}

The authors wish to thank Franziska Kluwe, Graduate Research Training Program PharMetrX and Department of Clinical Pharmacy and Biochemistry, Institute of Pharmacy, Freie Universitaet Berlin, for her support in the pharmacokinetics and nonlinear mixed-effects modelling analysis.

\section{Author Contributions}

FJ extended and implemented the mathematical models and did all numerical computations. ES and TF contributed to modelling, study design, and provided clinical data. KR contributed to data aquisition, mathematical modelling and developed the PK model for Ara-C. SS contributed to mathematical modelling, numerical approaches, and study design. All authors contributed to discussion of results and writing of the final paper.

\section{Data Availability}

Raw data contain patient-identifying information and are unsuitable for public deposition. Anonymized data sets can be obtained by contacting Prof. Dr. Sebastian Sager (sager@ovgu.de). 374 375 376 377 378 


\section{Supporting Information}

Appendix S1 Additional methods.

Table S1 Terminology and potentially confusing synonyms. Expressions that are also used in the manuscript are in italic.

392

Table S2 Comparison of model predictions for low-dose treatment schedules. As in Table 3 predicted values for different treatment schedules are shown, based on underlying mathematical models M1-M12. Shown are the values of median, standard deviation, minimum and maximum (in brackets) for two low-dose schedules. Both assume a constant administration of drugs throughout days 1 to 5 , with either $100 \mathrm{mg} / \mathrm{m}^{2}$ or $400 \mathrm{mg} / \mathrm{m}^{2}$ body surface. We do not have clinical data to compare these predictions, but they give additional insight on the possibility to discriminate models M1-M12 and a general trend showing that independent of the modelling assumptions both low-dose schedules result in slower white blood cell recovery and lower nadirs than D135 or D123. This has been clinically observed [3]. (Two further personalised cycles were excluded because for some models no recovery after chemotherapy was observed)

Table S3 Model constants, patient-specific constants, and units of model parameters. The values were used to obtain personalised mathematical models. The constants were determined from published data [26] and applied to all patients. To shorten notation we also used $c_{V}=\frac{1}{V_{c} M M_{\text {cyt }}}$. The patient-specific infusion times and dosages that define a treatment schedule were modified for simulation and optimisation of different schedules. The range shows minimum and maximum values of all considered data in the clinical study.

Table S4 Objectives (final objective function values from FOCEi method (OBJ), population predicted $t_{\text {rec }}^{123}$ and $t_{\text {rec }}^{135}$ values), parameter and coefficient of variation (CV) estimates with relative standard errors (RSE) from nonlinear mixed-effects modelling of models M3 and M10 with initial condition approach I1 (with I1).

Figure S1 Goodness-of-fit plot for all but three (because of WBC counts greater 1) measured and calculated $t_{r e c}$ values for model M10. The measured $t_{\text {rec }}$ values are slightly higher due to the coarser measurement grid.

Figure S2 Cross-validation of predicted white blood cell (WBC) counts from personalised models (PMs) M1-M12 and measured WBC counts for six patients treated with D135. All but one cycle were used for personalisation and the last cycle for cross-validation. For patients (a)-(e) the PMs can predict the WBC count decrease after Ara-C administration in the last cycle where models M10 and M12 have a slower WBC recovery than M5. For patient (f) the WBC recovery from the PMs starts to early compared to the measured WBC counts.

Figure S3 Cross-validation of predicted white blood cell (WBC) counts from personalised models (PMs) M1-M12 and measured WBC counts for five patients (a)-(e) treated with d135 and one patient (f) treated with D12. The PMs provide good predictions for patient (a) and (f) but show mismatches in recovery times and nadir values for patients (b)-(e). 
Figure S4 Comparing personalised mathematical models (PMs) M5 and M10 for D123 and D135 schedules (data set I).

Figure S5 Comparing personalised mathematical models (PMs) M5 and M10 for D123 and D135 schedules (data set II).

Figure S6 Comparing personalised mathematical models (PMs) M10 and M12 for D123 and D135 schedules (data set I).

Figure S7 Simulations of different pharmacokinetic models, Ara-C concentration measurements and inter-individual variability.

Figure S8 Visual predictive checks (VPCs), derived by 1000 simulations, for leukocytes $[G / L]$ versus time $[$ days $]$ starting with the first measurement before dosing for model M3 (a) and M10 (with I1) (b). Blue circles are the measured WBC counts of 23 AML patients described in section Clinical Data \& Personalisation. One measurement was taken at timepoint 88.98 [days] with the value $7.18[\mathrm{G} / \mathrm{L}]$ which is not shown in the VPCs. Red lines show the median (solid) and 5th and 95th percentiles (dashed) of measurements. The shaded areas represent the $90 \%$ confidence intervals around the 5th (blue), 50th (red) and 95th (blue) simulated percentiles of the model predictions. Regarding the VPCs, model M3 and M10 have an almost equivalent prediction accuracy. The $50 \%$ percentiles of measurements and model predictions perfectly overlap, thus supporting our individually based results from Table 2 The same applies to the start of the $5 \%$ and $95 \%$ percentiles until the nadir. After the nadir the $5 \%$ and $95 \%$ percentiles of the model predictions recover slightly faster/slower compared to the measurements. At day 30 the percentiles of measurements and model predictions coincide again.

Figure S9 Simulations of different pharmacokinetic models, Ara-C concentration measurements and inter-individual variability.

\section{References}

1. Malka R, Wolach B, Gavrieli R, Shochat E, Rom-Kedar V. Evidence for bistable bacteria-neutrophil interaction and its clinical implications. J Clin Invest. $2012 ; 122(8): 3002-3011$.

2. Döhner H, Estey E, Grimwade D, Amadori S, Appelbaum FR, Buchner T, et al Diagnosis and management of AML in adults: 2017 ELN recommendations from an international expert panel. Blood. 2017;129(4):424-447.

3. Mayer RJ, Davis RB, Schiffer CA, Berg DT, Powell BL, Schulman P, et al. Intensive postremission chemotherapy in adults with acute myeloid leukemia. Cancer and Leukemia Group B. N Engl J Med. 1994;331(14):896-903.

4. Wallin JE, Friberg LE, Karlsson MO. Model-Based Neutrophil-Guided Dose Adaptation in Chemotherapy: Evaluation of Predicted Outcome with Different Types and Amounts of Information. Basic Clin Pharmacol Toxicol. 2010;106(3):234-242.

5. Jost F, Sager S, Le TTT. A Feedback Optimal Control Algorithm with Optimal Measurement Time Points. Processes. 2017;5(10):1-19. 
6. Friberg LE, Henningsson A, Maas H, Nguyen L, Karlsson MO. Model of Chemotherapy-Induced Myelosuppression with Parameter Consistency Across Drugs. J Clin Oncol. 2002;20:4713-4721.

7. Quartino A, Friberg L, Karlsson M. A simultaneous analysis of the time-course of leukocytes and neutrophils following docetaxel administration using a semi-mechanistic myelosuppression model. Invest New Drugs. 2012;30:833-845.

8. Krogh-Madsen M, Bender B, Jensen MK, Nielsen OJ, Friberg LE, Honoré PH. Population pharmacokinetics of cytarabine, etoposide, and daunorubicin in the treatment for acute myeloid leukemia. Cancer chemother and Pharmacol. $2012 ; 69(5): 1155-1163$.

9. Pefani E, Panoskaltsis N, Mantalaris A, Georgiadis MC, Pistikopoulos EN. Chemotherapy drug scheduling for the induction treatment of patients with acute myeloid leukemia. IEEE Trans Biomed Eng. 2014;61(7):2049-2056.

10. Henrich A, Joerger M, Kraff S, Jaehde U, Huisinga W, Kloft C, et al. Semimechanistic Bone Marrow Exhaustion Pharmacokinetic/Pharmacodynamic Model for Chemotherapy-Induced Cumulative Neutropenia. J Pharmacol Exp Ther. 2017;362(2):347-358.

11. Mangas-Sanjuan V, Buil-Bruna N, Garrido MJ, Soto E, Trocóniz IF. Semimechanistic cell-cycle type-based pharmacokinetic/pharmacodynamic model of chemotherapy-induced neutropenic effects of diflomotecan under different dosing schedules. J Pharmacol Exp Ther. 2015;354(1):55-64.

12. Netterberg I, Nielsen EI, Friberg LE, Karlsson MO. Model-based prediction of myelosuppression and recovery based on frequent neutrophil monitoring. Cancer Chemother Pharmacol. 2017; p. 1-11.

13. Löwenberg B, Pabst T, Vellenga E, van Putten W, Schouten HC, Graux C, et al. Cytarabine dose for acute myeloid leukemia. N Engl J Med. 2011;364(11):1027-36. doi:10.1056/NEJMoa1010222.

14. Perry MC, Doll DC, Freter CE. Chemotherapy source book. Wolters Kluwer Health/Lippincott Williams \& Wilkins, Philadelphia; 2012.

15. Plunkett W, Liliemark JO, Adams TM, Nowak B, Estey E, Kantarjian H, et al. Saturation of $1-\beta$-D-arabinofuranosylcytosine 5 -triphosphate accumulation in leukemia cells during high-dose $1-\beta$-D-arabinofuranosylcytosine therapy. Cancer Res. 1987;47(11):3005-3011.

16. Li Z, Guo JR, Chen QQ, Wang CY, Zhang WJ, Yao MC, et al. Exploring the Antitumor Mechanism of High-Dose Cytarabine through the Metabolic Perturbations of Ribonucleotide and Deoxyribonucleotide in Human Promyelocytic Leukemia HL-60 Cells. Molecules. 2017;22(3):499.

17. Herold N, Rudd SG, Ljungblad L, Sanjiv K, Myrberg IH, Paulin CB, et al. Targeting SAMHD1 with the Vpx protein to improve cytarabine therapy for hematological malignancies. J Nat Med. 2017;23(2):256-263.

18. Clairambault J. Modelling physiological and pharmacological control on cell proliferation to optimise cancer treatments. Math Model Nat Phenom. $2009 ; 4(3): 12-67$. 
19. Craig M. Towards Quantitative Systems Pharmacology Models of Chemotherapy-Induced Neutropenia. CPT Pharmacometrics Syst Pharmacol. $2017 ;$

20. Wang W, Stiehl T, Raffel S, Hoang VT, Hoffmann I, Poisa-Beiro L, et al. Reduced hematopoietic stem cell frequency predicts outcome in acute myeloid leukemia. Haematologica. 2017;102(9):1567-1577.

21. Kloft C, Wallin J, Henningsson A, Chatelut E, Karlsson MO. Population pharmacokinetic-pharmacodynamic model for neutropenia with patient subgroup identification: comparison across anticancer drugs. Clin Cancer Res. 2006;12(18):5481-5490.

22. Quartino A. Pharmacometric Models for Improved Prediction of Myelosuppression and Treatment Response in Oncology; 2011.

23. Pefani E, Panoskaltsis N, Mantalaris A, Georgiadis MC, Pistikopoulos EN. Design of optimal patient-specific chemotherapy protocols for the treatment of acute myeloid leukemia (AML). Comput Chem Eng. 2013;57:187-195.

24. Nock V. Pharmacometric modelling of processes in the haematopoietic system and blood. Freie Universität Berlin; 2013.

25. Rinke K, Jost F, Findeisen R, Fischer T, Bartsch R, Schalk E, et al. Parameter estimation for leukocyte dynamics after chemotherapy. In: Proceedings of the Foundations of Systems Biology in Engineering (FOSBE) Conference. vol. 49. Magdeburg, Germany. Elsevier; 2016. p. 44-49.

26. Kern W, Schleyer E, Unterhalt M, Wörmann B, Büchner T, Hiddemann W. High antileukemic activity of sequential high dose cytosine arabinoside and mitoxantrone in patients with refractory acute leukemias. Cancer. 1997;79(1):59-68.

27. Solans BP, Fleury A, Freiwald M, Fritsch H, Haug K, Trocóniz IF. Population Pharmacokinetics of Volasertib Administered in Patients with Acute Myeloid Leukaemia as a Single Agent or in Combination with Cytarabine. Clin Pharmacokinet. 2018;57(3):379-392.

28. Minami H, Sasaki Y, Saijo N, Ohtsu T, Fujii H, Igarashi T, et al. Indirect-response model for the time course of leukopenia with anticancer drugs. Clin Pharmacol Ther. 1998;64(5):511-521.

29. Derendorf H, Meibohm B. Modeling of pharmacokinetic/pharmacodynamic (PK/PD) relationships: concepts and perspectives. Pharm Res. 1999;16(2):176-185.

30. Jaramillo S, Benner A, Krauter J, Martin H, Kindler T, Bentz M, et al. Condensed versus standard schedule of high-dose cytarabine consolidation therapy with pegfilgrastim growth factor support in acute myeloid leukemia. Blood Cancer J. 2017;7(5):e564.

31. Quartino A, Karlsson M, Lindman H, Friberg L. Characterization of endogenous G-CSF and the inverse correlation to chemotherapy-induced neutropenia in patients with breast cancer using population modeling. Pharm Res. 2014;31(12):3390-3403.

32. Andersson J. A General-Purpose Software Framework for Dynamic Optimization [PhD thesis]. Arenberg Doctoral School, KU Leuven; 2013. 
33. Hoffbrand AV, Higgs DR, Keeling DM, Mehta AB. Postgraduate Haematology. John Wiley \& Sons; 2016.

34. Takatani H, Soda H, Fukuda M, Watanabe M, Kinoshita A, Nakamura T, et al. Levels of recombinant human granulocyte colony-stimulating factor in serum are inversely correlated with circulating neutrophil counts. Antimicrob Agents Chemother. 1996;40(4):988-991.

35. Savic RM, Jonker DM, Kerbusch T, Karlsson MO. Implementation of a transit compartment model for describing drug absorption in pharmacokinetic studies. J Pharmacokinet Pharmacodyn. 2007;34(5):711-726.

36. Bock HG, Körkel S, Schlöder JP. In: Bock HG, Carraro T, Jäger W, Körkel S, Rannacher R, Schlöder JP, editors. Parameter Estimation and Optimum Experimental Design for Differential Equation Models. Heidelberg: Springer; 2013. p. $1-30$. 